SUGDEN: I agree that the situation is likely to be more complex in lowland peripheral areas of the ice sheet, especially because in areas of soft beds the underlying till may deform along with the glacier.

\title{
REFERENCE
}

Sugden, D. E. 1978. Glacial erosion by the Laurentide ice sheet. Fournal of Glaciology, Vol. 2 1, No. 83, p. 367-91.

\section{CHARACTERIZING THE GLACIER BED USING A RADIO-EGHO TECHNIQUE}

\author{
By C. S. M. Doake \\ (British Antarctic Survey, Natural Environment Research Council, Madingley Road, \\ Cambridge $\mathrm{CB}_{3}$ oET, England)
}

ABstract. If glacier ice is assumed to be a uniaxial birefringent material then two parameters are needed for describing the orientation behaviour of the polarization ellipse of a radio signal returned from the glacier bed. These are $\delta$, the relative phase between the ordinary and extraordinary wave, and $r$, the ratio of their attenuation coefficients (Woodruff and Doake, 1979). If it is assumed that the attenuation in ice is isotropic, then a value of $r$ other than unity will be due to anisotropic reflection coefficients at the glacier bed. A simple way of measuring $r$ separately from $\delta$ could then be used to characterize the reflecting surface.

Defining a rectangular coordinate system in which the wave propagates in the $z$ direction, the effective optic axis lies in the $z y$ plane, a linear transmitted wave of strength $E_{0}$ is at an angle $\alpha$ to the $x$ axis then the powers received in linear aerials aligned along the $x^{\prime} y^{\prime}$ axes of a rectangular coordinate system at an angle $\theta$ to the $x y$ system are given by

$P_{x}=E_{0}{ }^{2} A_{x^{2}},\left\{\cos ^{2} \alpha \cos ^{2} \theta+r^{2} \sin ^{2} \alpha \sin ^{2} \theta+2 r \sin \alpha \cos \alpha \sin \theta \cos \theta \cos \delta\right\}$,

$P_{y}=E_{0}^{2} A_{x}^{2}\left\{\cos ^{2} \alpha \sin ^{2} \theta+r^{2} \sin ^{2} \alpha \cos ^{2} \theta-2 r \sin \alpha \cos \alpha \sin \theta \cos \theta \cos \delta\right\}$,

where $A_{x}$ and $A_{y}$ are the attenuation coefficients in the $x$ and $y$ directions and $r=A_{y} \mid A_{x}$.

By adding the powers in the two aerials the total received power is

$$
P=P_{x},+P_{y},=E_{0}{ }^{2} A_{x}{ }^{2}\left(\cos ^{2} \alpha+r^{2} \sin ^{2} \alpha\right),
$$

which is independent of $\theta$, the azimuth of the receiving aerials. This is a well-known result in radar theory and practice. However, because of the dependence on $\alpha$, by rotating the transmitting aerial a value for $r$ can be found from the amplitude of the variation in the total power

$$
\begin{array}{rlrl}
r^{2} & =\frac{P_{\max }}{P_{\min }}, & r>\mathrm{I}, \\
r^{2}=\frac{P_{\min }}{P_{\max }}, & r<\mathrm{I} .
\end{array}
$$

The choice of the value for $r$ arises because of a $90^{\circ}$ ambiguity in the direction of the optic axis which can only be resolved by the full procedure of rotating both aerials.

\section{REFERENCE}

Woodruff, A. H. W., and Doake, C. S. M. 1979. Depolarization of radio waves can distinguish between floating and grounded ice sheets. Journal of Glaciology, Vol. 23, No. 89, p. 223-32. 